\title{
QS能
}

\section{ACUERDO UNIÓN EUROPEA-MERCOSUR: UNA REVISIÓN DE LOS EFECTOS ECONÓMICOS PREVISTOS}

Este trabajo realiza una revisión de la literatura empírica que ha estudiado el proceso negociador para alcanzar un acuerdo de libre comercio entre la Unión Europea y los países de Mercosur (Argentina, Brasil, Paraguay y Uruguay). Esta revisión ubica esta literatura en la perspectiva de todo el largo proceso negociador comenzado en 1992 y culminado con el Acuerdo de Asociación Estratégica de 2019. Se revisan los resultados cuantitativos de los estudios más próximos al acuerdo final y se muestra que este no permite alcanzar todas las ganancias que se derivan de la ventaja comparativa de España en manufacturas y servicios, y de Mercosur en productos agrarios.

Palabras clave: integración económica, modelos de equilibrio general aplicado, ecuación de gravedad, barreras arancelarias, barreras no arancelarias, ventaja comparativa.

Clasificación JEL: D58, F14, F15.

\section{Introducción}

El proceso negociador del Acuerdo de Libre Comercio entre la Unión Europea y el Mercado Común del Sur, conocido como Mercosur, ha tenido una larga andadura. El bloque signatario de Mercosur lo conforman Argentina, Brasil, Paraguay y Uruguay. En estos momentos existe ya un documento aceptado por las autoridades negociadoras de las dos partes, pero cuya entrada en vigor no se ha producido. Los objetivos de este trabajo se centran en presentar

\footnotetext{
* Departamento de Economía e INARBE. Universidad Pública de Navarra.

Versión de febrero de 2021.

DOI: https:/doi.org/10.32796/bice.2021.3133.7169
}

los estudios que han tratado de cuantificar los efectos económicos a lo largo del proceso negociador y profundizar especialmente en los estudios que abordan el último documento negociado, que ya ofrecen una aproximación cercana a los cambios que pueden acontecer, especialmente en la economía española, en el marco de su ventaja comparativa. Se mostrará cómo varios de estos estudios identifican a España como uno de los países en los que mayores efectos cuantitativos se pueden producir, en el contexto de la Unión Europea.

La estructura de este trabajo consta del apartado 2, en el que se presenta cómo el proceso negociador ha ido dando pie a distintos trabajos de investigación. A continuación, el $\triangleright$ 
apartado 3 esquematiza los estudios empíricos existentes con base en algunas de las características principales de los mismos. El apartado 4 profundiza en los resultados de los trabajos que analizan los efectos económicos del último acuerdo alcanzado vinculándolos a la ventaja comparativa española. Por último, el apartado 5 presenta unas conclusiones.

\section{Estudios económicos durante el proceso de negociación}

\subsection{Los albores de la negociación: 1992-2004}

La Comisión Europea y los cuatro países de Mercosur firmaron un primer acuerdo de cooperación interregional el 29 de mayo de 1992, poco más de un año después del establecimiento de Mercosur (creado por el Tratado de Asunción del 26 de marzo de 1991). La estructura institucional de Mercosur quedó establecida el 17 de diciembre de 1994, con la firma del Protocolo de Ouro Preto. Con este marco institucional, el 15 de diciembre de 1995 la UE y Mercosur firmaban en Madrid un Acuerdo Marco Interregional de Cooperación que entraría en vigor el 1 de julio de 1999. A raíz de este acuerdo, el Consejo Europeo encargó a la Comisión Europea la negociación de un acuerdo de asociación entre la UE y Mercosur. Este futuro acuerdo, como señala Grieger (2019: 6-8), debería sustentarse en tres pilares: una asociación en materia política y de seguridad, la cooperación económica e institucional y la creación gradual de un área de libre comercio de bienes y servicios.

Las negociaciones formales comenzaron en abril de 2000 (para una explicación extensa de todo el proceso negociador hasta la consecución del acuerdo en 2019 véase Sanahuja y Rodríguez, 2019). En esas fechas estaban teniendo lugar en la Organización Mundial del Comercio las conversaciones vinculadas a la reunión ministerial de Doha (Qatar) y, en el contexto americano, se negociaba el fallido Tratado de Libre Comercio de las Américas, ALCA. La negociación coetánea de estos acuerdos diferentes provocó que la mayoría de los trabajos de investigación de la época incluyeran el estudio simultáneo de los efectos de estos procesos de negociación, sin posibilidad de distinguir los efectos de unos u otros acuerdos. Sin embargo, se publicaron trabajos que analizaban explícitamente por separado el área de libre comercio UE-Mercosur, como los de Harrison et al. (2003) y Diao et al. (2003). El primero de ellos ya asumía en el análisis uno de los aspectos clave en la cuantificación de los efectos económicos, como es el tratamiento especial a productos sensibles ante una liberalización comercial total. Así, Harrison et al. (2003) incluyen un escenario en el que no varían las barreras comerciales europeas a productos tales como arroz, cereales, carne, lácteos y azúcar. Este trato diferenciado entre tipos de bienes no es una constante en los trabajos posteriores, pero es una muestra de la complejidad del análisis, que, además, suele llevar aparejado el uso de instrumentos de política comercial diferentes a los aranceles. En este sentido, Harrison et al. (2003) ya introducían a través de equivalentes arancelarios una aproximación a estos instrumentos no arancelarios.

\subsection{La paralización de las negociaciones: 2004-2010}

Las negociaciones, no el diálogo, entre la UE y Mercosur se paralizaron en diciembre $D$ 
de 2004, a causa del desencuentro en relación con los niveles de liberalización del comercio que cada parte defendía (Burrel et al., 2011: 1719; Sanahuja y Rodríguez, 2019: 10-11). Entre otros aspectos, del lado de Mercosur se consideraba que la propuesta comunitaria exigía una gran liberalización industrial mientras se concedía una escasa liberalización agrícola. Del lado de la UE, se consideraban insuficientes los avances en la liberalización comercial de sectores como el textil, el calzado y el automóvil.

La paralización de las negociaciones se alargó hasta que, en 2009, se retomaron negociaciones informales y, ya en mayo de 2010, se volvieron a formalizar en la madrileña VI Cumbre Unión Europea-América Latina y el Caribe. La UE ya contaba con un número mayor de socios, lo que añadía complejidad, sobre todo, a la negociación en temas agrícolas. En ese periodo de impasse sin negociaciones Flôres y Watanuki (2008) realizaron un análisis de diferentes acuerdos comerciales bilaterales que en esos momentos Mercosur tenía en estudio, entre los que se encontraba el acuerdo con la UE, aunque el estudio consideraba una liberalización comercial general. Sin embargo, Boyer y Schuschny (2010) recogen en sus simulaciones un tratamiento diferenciado a varios productos sensibles que habían ido apareciendo en las negociaciones. La novedad en este tratamiento diferenciado está en que no solo se recogían limitaciones europeas a productos agrícolas (tales como arroz, carne, lácteos, bebidas y tabaco), sino también limitaciones de Mercosur a una larga lista de productos industriales europeos (como minerales, textiles, papel, productos químicos, productos metálicos, automóviles y sus partes, maquinaria y equipo, y electrónica).

La rechazada propuesta de 2004 de la UE y la contraoferta que Mercosur envió en 2006 dieron origen a diversos estudios que las abordaban. Además, con la falta de avances en la Ronda de Doha, los países tenían incentivos para avanzar en conversaciones de carácter bilateral. Entre estos estudios está el de Burrel et al. (2011), que analizaba de manera combinada el posible acuerdo UE-Mercosur con el cumplimiento del acuerdo de Doha. En este punto del proceso ya se planteaban aspectos vinculados a la noción de sostenibilidad. Así, en 2006 la Comisión Europea puso en marcha una evaluación inicial de impacto de sostenibilidad (Sustainability Impact Assessment, SIA) del acuerdo, materializada por Kirkpatrick et al. (2006). En 2009 se publicó el documento final del SIA (Kirkpatrick et al., 2009). Entre ambos informes se fueron publicando resultados parciales de carácter sectorial para la agricultura, el sector forestal y el automóvil. En 2010, el Parlamento Europeo dio su parecer sobre el SIA y constató que las ganancias económicas para la UE y para Mercosur existían, pero hacía hincapié en los costes medioambientales que el acuerdo podría generar. El mismo aspecto se había señalado anteriormente por la Comisión Europea. Este es un aspecto relevante que en el acuerdo de 2019 también se ha tenido en cuenta al integrar, por ejemplo, los compromisos de emisiones del Acuerdo de París y otras exigencias medioambientales.

El proyecto de acuerdo también se analiza en la evaluación de impacto económico (Economic Impact Assessment) encargada por la DG Trade de la UE a Copenhagen Economics (2011). Todos estos trabajos, posteriores a 2004, ya incluían simulaciones que iban más allá de la mera eliminación o reducción de aranceles, por ejemplo, incluyendo efectos sobre facilitación del comercio o la aplicación de contingentes arancelarios. 


\subsection{La última y larga fase de la negociación: 2010-2019}

Tras la reanudación formal de las negociaciones en 2010, estas volverían a paralizarse nuevamente en 2012 por los cambios políticos que estaban teniendo lugar entre los socios de Mercosur: los problemas políticos en Paraguay, que incluyeron la destitución del presidente Fernando Lugo, llevaron a suspender a este país como socio. También Venezuela se unió al bloque y los Gobiernos de Cristina Fernández de Kirchner en Argentina y Dilma Rousseff en Brasil orientaron sus preferencias hacia las relaciones Sur-Sur (Grieger, 2019). Los estudios encargados por la UE seguían apareciendo. Así, el trabajo de Boulanger et al. (2016) analizaba el efecto acumulativo de los acuerdos de libre comercio recientes en el sector agrícola de la UE, siendo Mercosur el que implicaba un mayor volumen de importaciones de los doce acuerdos que se recogen en el estudio, y el quinto en exportaciones. Este sector agrícola también fue estudiado a fondo en el marco del modelo CAPRI por los trabajos de Weissleder, Adenauer y Heckelei (2008), Piketty et al. (2009) y Burrell et al. (2011). Todo ello permitía entender la posición negociadora de la Comisión Europea al ofrecer preferencias a Mercosur hasta un volumen limitado a través de contingentes arancelarios en productos agrícolas.

En este contexto, también se fueron realizando otros trabajos fuera del ámbito de las instituciones comunitarias, pero estos no llegaban a simular un acuerdo de integración económica con la complejidad que se iba acotando en las negociaciones y que ya recogían los últimos trabajos arriba comentados. Entre estos trabajos no realizados explícitamente para las instituciones europeas están los de
Estrades (2012), Philippidis, Resano y Sanjuán (2014) y Sanguinet y Alvim (2020).

Después de un intercambio de nuevas ofertas de acceso a los mercados, formuladas a lo largo del año 2016, las negociaciones cobraron impulso hasta noviembre de 2017, cuando Mercosur entregó ya una propuesta integral de acuerdo. Sin embargo, la agricultura siguió siendo la mayor traba, y a finales de 2018 se habían logrado pocos avances con respecto a los principales intereses de la UE, tales como el sector del automóvil, los productos lácteos, los servicios marítimos y la protección de las indicaciones geográficas. Dado que el SIA de 2009 estaba desactualizado, se llevó a cabo uno nuevo, cuyo informe inicial (London School of Economics Consulting, 2018) proporcionaba información sobre los nuevos desarrollos en las relaciones comerciales UE-Mercosur. En su informe final (London School of Economics Consulting, 2020) se realizaban las estimaciones del impacto cualitativo y cuantitativo del potencial acuerdo. Lamentablemente, este estudio no ofrecía los resultados desagregados para la economía española.

A pesar de los desacuerdos a lo largo de todo el proceso de negociación, de los serios desafíos planteados al sistema de comercio multilateral por el aumento del unilateralismo y el proteccionismo - guerra comercial entre EE UU y China, Brexit...; véase Martínez Serrano (2020) para una perspectiva - se llegó a la formalización del Acuerdo de Asociación Estratégica el 28 de junio de 2019. Este acuerdo está a la espera de su ratificación definitiva por todos los países. Ad interim han ido apareciendo estudios realizados por instituciones de carácter nacional de los distintos Estados miembros de la UE que sí tratan de aproximar ya las cláusulas finales del acuerdo. Así, Timini y Viani (2020) realizan un modelo de gravedad $\triangleright$ 
con la economía española (y otras) desagregada, que es publicado por el Banco de España. Sinabell, Grübler y Reiter (2020) elaboran otro modelo de gravedad con especial análisis del caso austriaco, y Carrico et al. (2020) centran su estudio en los efectos para la economía neerlandesa.

\section{Características principales de los estudios empíricos del Acuerdo UE-Mercosur}

En general, los efectos estimados por todos los estudios comentados previamente son pequeños. Muchos de estos estudios analizan solo una eliminación total o parcial de los aranceles y, en ocasiones, la presencia de equivalentes arancelarios de barreras no arancelarias. Sin embargo, el Acuerdo de Asociación Estratégica del 28 de junio de 2019 no llega a un nivel de desarme arancelario total e incorpora otros aspectos más difíciles de modelizar. Por ello, y a la vista de cómo iban sucediéndose las negociaciones y como se ha comentado en la sección anterior, algunos de los trabajos realizados ya consideraban la hipótesis de incluir sectores sensibles, en los que previsiblemente no se iba a producir una liberalización comercial total, que sería la que daría potencialmente las mayores ganancias. Además, el acuerdo de 2019 establece un periodo de transición para el desmantelamiento arancelario que parece que llega hasta diez años para productos sensibles e incluso hasta quince para las importaciones de automóviles en Mercosur, lo cual hará menos visibles las ganancias, ya que estarán repartidas a lo largo del proceso temporal de desarme de barreras. Es relevante indicar, además, que algunos trabajos modelizan productos sensibles que son sometidos a cuotas arancelarias cuya existencia reduce el potencial de ganancias.

Más adelante se presentan los Cuadros 1 y 2 , que recogen las principales características de los diferentes estudios que se han comentado en la sección anterior. En el Cuadro 1 se recogen, en orden cronológico, los modelos de equilibrio general aplicado que han estudiado un acuerdo de libre comercio entre la UE y Mercosur. Para una explicación introductoria a estos modelos, véase Gómez Gómez-Plana (2005). Las características que se recogen en dicho cuadro son algunas características distintivas de los modelos de equilibrio general aplicado utilizados, la base de datos (y año) utilizada en el análisis, una descripción sumaria de las simulaciones efectuadas vinculadas al acuerdo y la desagregación geográfica planteada sobre la que cada trabajo ofrece sus resultados. Son los modelos más numerosos. En el Cuadro 2, de manera similar, se recoge la misma información con otras metodologías, como son el modelo de equilibrio parcial CAPRI y otros modelos basados en ecuaciones de gravedad que se han construido y aplicado al análisis de un acuerdo comercial entre la UE y Mercosur.

\section{Algunos resultados cuantitativos vinculados al acuerdo final UE-Mercosur}

En el marco de los modelos de ventaja comparativa, Mercosur tiene ventaja comparativa en el sector agrícola, mientras que la Unión Europea y España la tendrían en los sectores manufactureros y de servicios. El Cuadro 3, que muestra la estimación del índice de ventaja comparativa revelada para España y los cuatro socios de Mercosur, así lo corrobora, en $\triangleright$ 
CUADRO 2

OTRAS METODOLOGÍAS QUE ANALIZAN UN ACUERDO UE-MERCOSUR

\begin{tabular}{|c|c|c|c|c|}
\hline Referencia & Modelo & Datos & Escenarios & Desagregación geográfica \\
\hline $\begin{array}{l}\text { Weissleder, } \\
\text { Adenauer } \\
\text { y Heckelei (2008) }\end{array}$ & $\begin{array}{l}\text { Modelo de equilibrio } \\
\text { parcial CAPRI (sectores } \\
\text { agrícolas) }\end{array}$ & $\begin{array}{l}\text { Dos baseline } \\
2013\end{array}$ & $\begin{array}{l}\text { (1) Propuesta de la UE de } 2004 \\
\text { (2) Propuesta de la UE con } \\
\text { liberalización de otras regiones } \\
\text { (3) Liberalización total }\end{array}$ & $\begin{array}{l}\text { UE25, Argentina, Brasil, } \\
\text { Paraguay, Uruguay y } \\
\text { Venezuela }\end{array}$ \\
\hline $\begin{array}{l}\text { Piketty et al. } \\
\text { (2009) }\end{array}$ & $\begin{array}{l}\text { Modelo de equilibrio } \\
\text { parcial CAPRI (sectores } \\
\text { agrícolas) }\end{array}$ & $\begin{array}{l}\text { Baseline } \\
2013\end{array}$ & $\begin{array}{l}\text { (1) Propuesta de la UE de } 2004 \\
\text { (2) Propuesta de Mercosur de } 2006 \\
\text { (3) Liberalización total }\end{array}$ & Regiones EU NUTS 2 y Brasil \\
\hline $\begin{array}{l}\text { Burrell et al. } \\
(2011)\end{array}$ & $\begin{array}{l}\text { Modelo de equilibrio } \\
\text { parcial CAPRI (sectores } \\
\text { agrícolas) }\end{array}$ & $\begin{array}{l}\text { Baseline } \\
2010\end{array}$ & $\begin{array}{l}\text { (1) Propuesta de la UE de } 2004 \\
\text { (2) Propuesta de Mercosur de } 2006\end{array}$ & $\begin{array}{l}\text { UE por países, Argentina, } \\
\text { Brasil, Uruguay, Paraguay }\end{array}$ \\
\hline $\begin{array}{l}\text { Timini y Viani } \\
(2020)\end{array}$ & $\begin{array}{l}\text { Modelo de equilibrio } \\
\text { general de ecuación de } \\
\text { gravedad estructural } \\
\text { (Baier, Yotov y Zylkin, } \\
\text { 2019) }\end{array}$ & $1984-2015$ & $\begin{array}{l}\text { (1) Aplicación del acuerdo } \\
\text { (2) Un país de Mercosur no aplica el } \\
\text { acuerdo } \\
\text { (3) Efecto globalización } \\
\text { (4) Acuerdos homogéneos } \\
\text { (5) Mayor elasticidad de comercio }\end{array}$ & $\begin{array}{l}53 \text { países, incluyendo } \\
\text { separados Mercosur y } 17 \\
\text { países UE }\end{array}$ \\
\hline $\begin{array}{l}\text { Sinabell, Grübler y } \\
\text { Reiter (2020) }\end{array}$ & $\begin{array}{l}\text { Modelo de equilibrio } \\
\text { general de ecuación de } \\
\text { gravedad estructural } \\
\text { (Baier, Yotov y Zylkin, } \\
\text { 2019) }\end{array}$ & $1995-2017$ & $\begin{array}{l}\text { Eliminación de aranceles, profundidad } \\
\text { del acuerdo } 5 \text { sobre } 7 \text { (DESTA), } \\
\text { centralidad }\end{array}$ & $\begin{array}{l}\text { Casi } 200 \text { países, con UE27 y } \\
\text { Mercosur desagregados }\end{array}$ \\
\hline
\end{tabular}

general. El índice estimado, diseñado por Bebek (2011), mejora el tradicional índice de ventaja comparativa revelada de Balassa (1965) y tiene mejores propiedades que el de Yu, Cai y Leung (2009) 1 . Los datos utilizados para la realización del índice son los de GTAP 9, Aguiar et al. (2016), que son unos de los datos más habituales utilizados en los análisis que aquí se comentan (Cuadro 1) y, por tanto, están en la base de los resultados de las estimaciones que existen para cuantificar los efectos económicos del acuerdo entre la UE y Mercosur.

Las ventajas comparativas potencialmente serían tanto ricardiana (esto es, basada en las diferencias en productividad) como de tipo Heckscher-Ohlin-Samuelson (basada en las

\footnotetext{
1 Sin embargo, los resultados que se presentan en el Cuadro 3 dan el mismo patrón de ventaja comparativa que los que también se han obtenido con la estimación del índice de Yu, Cai y Leung (2009) con los mismos datos. La correlación para el caso estudiado es perfecta entre ambos índices, por lo que solo se presentan aquí los resultados para el índice estimado con la metodología de Bebek (2011). Yu, Cai y Leung (2009) ofrecen una amplia panorámica sobre el índice de ventaja comparativa revelada y sus propiedades.
}

dotaciones relativas de factores dada la intensidad relativa de los bienes). Por tanto, se podría predecir que el acuerdo de 2019 podría llevar a una mayor especialización de cada región en los bienes y servicios en los que cuentan con ventaja comparativa, lo que implicaría un ajuste en los niveles de actividad sectorial en cada región. Esto, en ausencia de un aumento de las posibilidades de producción, implicaría una expansión de la producción en los sectores con ventaja comparativa, pero una contracción en los sectores con desventaja comparativa. Por todo ello, y dado el perfil comercial de cada región, parece que la desviación de comercio sería muy baja. Estos resultados son los que, en general, han ido obteniéndose en los trabajos que han simulado el acuerdo, especialmente en aquellos que limitan las simulaciones más al ámbito arancelario sin sectores sensibles. De hecho, hay aspectos adicionales como los indicados por Baltensperger y Dadush (2019) que revelan que se $D$ 
CUADRO 3

ÍNDICES DE VENTAJA COMPARATIVA REVELADA

\begin{tabular}{|c|c|c|c|c|c|}
\hline Bienes/servicios & Argentina & Brasil & Paraguay & Uruguay & España \\
\hline Arroz con cáscara & 0,021497 & $-0,158032$ & 0,109879 & 0,084469 & $-0,057814$ \\
\hline Trigo & 3,406937 & $-3,293114$ & 0,167503 & 0,539112 & $-0,820438$ \\
\hline Cereales ncop & 0,323588 & $-0,442636$ & 0,454347 & $-0,088611$ & $-0,246689$ \\
\hline Vegetales, frutas, nuts & 1,685934 & $-1,373345$ & $-0,089118$ & $-0,061392$ & $-0,162079$ \\
\hline Semillas oleaginosas & $-1,801504$ & 1,816585 & 0,908779 & $-0,245536$ & $-0,678324$ \\
\hline Caña de azúcar y remolacha azucarera & $-0,000005$ & $-0,000002$ & $-0,000001$ & $-0,000001$ & 0,000009 \\
\hline Fibras naturales & 0,054076 & $-0,025905$ & $-0,001848$ & $-0,007238$ & $-0,019086$ \\
\hline Cosechas ncop & $-0,560047$ & 0,908927 & $-0,035022$ & $-0,078738$ & $-0,235120$ \\
\hline Bovino, ovino, caprino, caballos & $-0,001543$ & $-0,016771$ & $-0,001419$ & 0,024213 & $-0,004480$ \\
\hline Otros productos animales ncop & $-0,062650$ & 0,020525 & 0,000959 & 0,068401 & $-0,027235$ \\
\hline Leche & $-0,000176$ & 0,000011 & 0,000001 & $-0,000014$ & 0,000178 \\
\hline Lana y seda & $-0,002447$ & 0,022993 & $-0,003963$ & $-0,004345$ & $-0,012238$ \\
\hline Productos forestales & $-0,019448$ & 0,004107 & 0,008875 & 0,011789 & $-0,005324$ \\
\hline Pesca & 0,033739 & $-0,031262$ & $-0,002067$ & 0,001816 & $-0,002226$ \\
\hline Carbón & $-0,000043$ & $-0,000059$ & $-0,000004$ & $-0,000005$ & 0,000112 \\
\hline Petróleo & $-0,286466$ & 0,443286 & $-0,025168$ & $-0,034796$ & $-0,096856$ \\
\hline Gas & 0,053344 & $-0,038052$ & $-0,002454$ & $-0,003393$ & $-0,009444$ \\
\hline Minerales ncop & $-2,642079$ & 4,105271 & $-0,250628$ & $-0,378368$ & $-0,834197$ \\
\hline Productos cárnicos de bovino & $-0,083539$ & $-0,653169$ & 0,152907 & 0,779581 & $-0,195780$ \\
\hline Otros productos cárnicos ncop & $-0,474228$ & 0,709561 & $-0,046411$ & $-0,059007$ & $-0,129914$ \\
\hline Aceites vegetales y grasas & 1,640301 & $-1,433512$ & 0,156102 & $-0,029184$ & $-0,333707$ \\
\hline Productos lácteos & 0,731240 & $-1,114867$ & $-0,074315$ & 0,727350 & $-0,269408$ \\
\hline Arroz procesado & 0,085826 & $-0,332206$ & 0,115015 & 0,216285 & $-0,084920$ \\
\hline Azúcar & $-0,395386$ & 0,605813 & $-0,029687$ & $-0,047937$ & $-0,132802$ \\
\hline Otros alimentos ncop & 3,295666 & $-2,402084$ & $-0,143921$ & 0,086564 & $-0,836226$ \\
\hline Bebidas y tabaco & 0,504057 & $-1,034283$ & $-0,074127$ & 0,802482 & $-0,198129$ \\
\hline Textiles & $-0,416285$ & 0,260012 & 0,152695 & 0,126793 & $-0,123216$ \\
\hline Tejidos & $-0,127509$ & $-0,221694$ & 0,117252 & 0,066524 & 0,165426 \\
\hline Productos de cuero & $-0,498061$ & 0,521705 & 0,094018 & 0,037183 & $-0,154845$ \\
\hline Productos de la madera & $-0,473656$ & 0,136817 & 0,064503 & 0,359678 & $-0,087342$ \\
\hline Productos de papel, artes gráficas & $-0,578229$ & 0,383280 & $-0,103942$ & 0,205392 & 0,093500 \\
\hline Derivados del carbón y del petróleo & 2,103322 & 0,563135 & $-0,566445$ & $-0,419527$ & $-1,680484$ \\
\hline Productos químicos, caucho y plástico & 1,286587 & $-2,269769$ & $-0,813145$ & 0,859596 & 0,936730 \\
\hline Productos minerales ncop & $-0,376836$ & 0,218660 & $-0,036645$ & 0,032315 & 0,162506 \\
\hline Metales férricos & $-1,230524$ & 1,573277 & $-0,128858$ & $-0,049938$ & $-0,163956$ \\
\hline Metales ncop & 0,087704 & 0,359073 & 0,019309 & $-0,130320$ & $-0,335765$ \\
\hline Productos metálicos & $-0,568098$ & 0,445900 & $-0,107574$ & $-0,122296$ & 0,352068 \\
\hline Vehículos de motor y sus partes & 6,526111 & 3,267718 & $-2,038470$ & $-1,657916$ & $-6,097442$ \\
\hline Equipo de transporte ncop & $-1,075416$ & 0,331246 & $-0,118327$ & $-0,162310$ & 1,024808 \\
\hline Equipo electrónico & $-0,494365$ & 0,593952 & $-0,056708$ & $-0,065176$ & 0,022297 \\
\hline Maquinaria y equipo ncop & $-3,779108$ & 3,268035 & $-0,544804$ & $-0,650919$ & 1,706795 \\
\hline
\end{tabular}


CUADRO 3 (cont.)

ÍNDICES DE VENTAJA COMPARATIVA REVELADA

\begin{tabular}{|l|c|c|c|c|c|}
\hline \multicolumn{1}{|c|}{ Bienes/servicios } & Argentina & Brasil & Paraguay & Uruguay & España \\
\hline Manufacturas ncop & $-0,177485$ & 0,073556 & $-0,014173$ & $-0,020976$ & 0,139078 \\
Electricidad & $-1,347558$ & $-1,825397$ & 3,261622 & $-0,170820$ & 0,082153 \\
Distribución y manufactura del gas & 0,000217 & $-0,000155$ & $-0,000010$ & $-0,000014$ & $-0,000038$ \\
Agua & $-0,005549$ & $-0,006201$ & $-0,000648$ & $-0,000301$ & 0,012701 \\
Construcción & $-0,026755$ & $-0,044297$ & $-0,002039$ & $-0,003668$ & 0,076758 \\
Comercio & $-0,672436$ & $-0,915005$ & $-0,052610$ & $-0,070743$ & 1,710794 \\
Transporte ncop & $-0,479471$ & $-0,699474$ & $-0,054315$ & $-0,027184$ & 1,260444 \\
Transporte acuático & $-0,284247$ & $-0,252837$ & $-0,033136$ & $-0,044413$ & 0,614633 \\
Transporte aéreo & $-0,325921$ & $-0,571572$ & $-0,046868$ & $-0,067384$ & 1,011746 \\
Comunicación & $-0,037782$ & $-0,088583$ & $-0,006005$ & $-0,006055$ & 0,138425 \\
Servicios financieros ncop & $-0,202921$ & $-0,111997$ & $-0,018443$ & $-0,015600$ & 0,348960 \\
Seguros & $-0,066692$ & $-0,055780$ & $-0,001552$ & $-0,006970$ & 0,130994 \\
Servicios a las empresas ncop & $-1,600563$ & $-0,042969$ & $-0,182638$ & $-0,225608$ & 2,051777 \\
Servicios recreativos y otros servicios & $-0,389239$ & $-0,791770$ & $-0,052288$ & $-0,055807$ & 1,289103 \\
Administración Pública, defensa, educación y salud & $-0,275874$ & $-0,386652$ & $-0,023973$ & $-0,017034$ & 0,703533 \\
\hline Ncop: no clasificado en otra parte. & & & \\
Fuente: elaboración propia a partir de la base de datos GTAP9, Aguiar et al. (2016). &
\end{tabular}

obtendrían mayores ganancias si la liberalización parcial acordada de los flujos comerciales llevara a reformas que implicaran la mejora a largo plazo de la productividad en el sector agrícola de la UE y en el sector manufacturero de Mercosur, por el ajuste en las ventajas comparativas a causa del comercio.

Reviste interés conocer especialmente los modelos que abordan el Acuerdo de Asociación Estratégica del 28 de junio de 2019. Estos se recogen en el Cuadro 4. Utilizan dos metodologías diferentes: tres son modelos de equilibrio general aplicado y dos corresponden a modelos de gravedad. En este Cuadro 4 se indica si el modelo cuantifica los efectos económicos separadamente para España, o si España está agregada al conjunto de países de la UE o a un subconjunto. Además, se indica el nivel de desagregación por sectores de actividad económica y las variables estudiadas (tanto las variables agregadas como aquellas que se presentan por sectores).
A continuación, el Cuadro 5 se centra en algunos resultados cuantitativos macroeconómicos de los trabajos del Cuadro 4. Los resultados no son en muchos casos directamente comparables, pero hay algunas características que se pueden resaltar, y que se comentan a continuación. Nótese que se indica si el resultado cuantitativo se refiere a España en particular (cuando está disponible ese dato) o al área de la UE en la que España está integrada en el trabajo.

Los resultados sobre el PIB nos muestran que los resultados, tanto para la UE en su conjunto como para España en particular, serían cuantitativamente muy pequeños. Solo existe una estimación específica para España, realizada por Sinabell, Grübler y Reiter (2020), que prevé un ligerísimo aumento del $0,081 \%$ del PIB. Por otro lado, Sanguinet y Alvim (2020) presentan unos resultados cuantitativos sustancialmente mayores para el conjunto de la UE, pero hay que tener en cuenta que su $\triangleright$ 
CUADRO 4

MODELOS DE EQUILIBRIO GENERAL APLICADO QUE ANALIZAN EL ACUERDO UE-MERCOSUR DE 2019

\begin{tabular}{|c|c|c|c|c|}
\hline Referencia & Modelo & España & Sectores & Variables estudiadas \\
\hline $\begin{array}{l}\text { London } \\
\text { School of } \\
\text { Economics } \\
\text { Consulting } \\
(2018,2020)\end{array}$ & $\begin{array}{l}\text { Modelo de } \\
\text { equilibrio } \\
\text { general } \\
\text { aplicado }\end{array}$ & $\begin{array}{l}\text { Agregada } \\
\text { en UE-28 }\end{array}$ & $\begin{array}{l}31 \text { sectores: } 12 \text { agrícolas y ganaderos, } 4 \\
\text { extractivos, } 9 \text { manufactureros, } 6 \text { servicios }\end{array}$ & $\begin{array}{l}\text { Agregadas: PIB, inversión, } \\
\text { bienestar, importaciones, } \\
\text { exportaciones, precios, } \\
\text { salarios reales calificados y no } \\
\text { calificados, emisiones CO2, } \\
\mathrm{CH} 4, \mathrm{~N} 2 \mathrm{O} \text {. } \\
\text { Sectoriales: exportaciones, } \\
\text { importaciones, output, } \\
\text { emisiones, empleo, consumo } \\
\text { privado }\end{array}$ \\
\hline $\begin{array}{l}\text { Sanguinet y } \\
\text { Alvim (2020) }\end{array}$ & $\begin{array}{l}\text { Modelo de } \\
\text { equilibrio } \\
\text { general } \\
\text { aplicado } \\
\text { GTAP }\end{array}$ & $\begin{array}{l}\text { Agregada } \\
\text { en UE-27 } \\
\text { (sin Reino } \\
\text { Unido) }\end{array}$ & $\begin{array}{l}9 \text { sectores: cereales, carnes, extracción, } \\
\text { alimentos, textil, manufacturas, utilities y } \\
\text { construcción, transportes y comunicaciones, otros } \\
\text { servicios }\end{array}$ & $\begin{array}{l}\text { Agregadas: variaciones } \\
\text { equivalentes, PIB. } \\
\text { Sectoriales: exportaciones, } \\
\text { exportaciones bilaterales, } \\
\text { importaciones bilaterales, } \\
\text { precios }\end{array}$ \\
\hline $\begin{array}{l}\text { Carrico et al. } \\
(2020)\end{array}$ & $\begin{array}{l}\text { Modelo de } \\
\text { equilibrio } \\
\text { general } \\
\text { aplicado } \\
\text { MAGNET }\end{array}$ & $\begin{array}{l}\text { Agregada } \\
\text { en UE-27 } \\
\text { (con Reino } \\
\text { Unido, sin } \\
\text { Países } \\
\text { Bajos) }\end{array}$ & $\begin{array}{l}18 \text { sectores (no presentan todos): frutas y vegetales, } \\
\text { ternera, pollo, cerdo y otras carnes, feed, lácteos, } \\
\text { azúcar, química, farmacéutica, etanol, manufacturas } \\
\text { ligeras, maquinaria, automóviles, otra maquinaria de } \\
\text { transporte, manufactura pesada, transporte por agua, } \\
\text { comercio, servicios profesionales }\end{array}$ & $\begin{array}{l}\text { Sectoriales: output, salario real, } \\
\text { exportaciones, importaciones, } \\
\text { saldo comercial }\end{array}$ \\
\hline $\begin{array}{l}\text { Timini y Viani } \\
(2020)\end{array}$ & $\begin{array}{l}\text { Ecuación de } \\
\text { gravedad }\end{array}$ & Sí & No & $\begin{array}{l}\text { Agregadas: exportaciones, } \\
\text { importaciones, salario nominal, } \\
\text { salario real, precios, comercio } \\
\text { interior }\end{array}$ \\
\hline $\begin{array}{l}\text { Sinabell, } \\
\text { Grübler y } \\
\text { Reiter (2020) }\end{array}$ & $\begin{array}{l}\text { Ecuación de } \\
\text { gravedad }\end{array}$ & Sí & No & $\begin{array}{l}\text { Agregadas: output, } \\
\text { exportaciones, empleo, PIB }\end{array}$ \\
\hline
\end{tabular}

CUADRO 5

RESULTADOS EMPÍRICOS: MODELOS DE EQUILIBRIO GENERAL APLICADO QUE ANALIZAN EL ACUERDO UE-MERCOSUR DE 2019

\begin{tabular}{|c|c|c|c|c|c|c|c|}
\hline Referencia & Escenario & Zona & PIB & Bienestar & Salario real & Importaciones & Exportaciones \\
\hline \multirow{2}{*}{$\begin{array}{l}\text { London School of Economics } \\
\text { Consulting }(2018,2020)\end{array}$} & Conservador & UE28 & $0,1 \%$ & $6,3^{1}$ & $0,2 \%{ }^{2}$ & $0,9 \%$ & $0,4 \%$ \\
\hline & Ambicioso & UE28 & $0,1 \%$ & $8,6^{1}$ & $0,3 \%^{2}$ & $1,1 \%$ & $0,6 \%$ \\
\hline \multirow{2}{*}{ Sanguinet y Alvim (2020) } & Sin Brexit & UE27 & $10,05 \%$ & $5,4^{3}$ & & & \\
\hline & Con Brexit & UE27 & $10,06 \%$ & $5,1^{3}$ & & & \\
\hline Carrico et al. (2020) & Único & UE274 & $0,02 \%$ & & {$[-0,7 \%-1,5 \%]^{5}$} & {$[0 \%-215 \%]^{5}$} & {$[-5 \%-225 \%]^{5}$} \\
\hline \multirow{2}{*}{ Timini y Viani (2020) } & & España & & & $0,10 \%$ & $1,29 \%$ & $1,44 \%$ \\
\hline & & UE16 6 & & & {$[0,01 \%-0,39 \%]^{6}$} & {$[-0,06 \%-1,29 \%]^{6}$} & {$[-0,06 \%-1,44 \%]^{6}$} \\
\hline Sinabell, Grübler y Reiter & & España & $0,081 \%$ & & & & $4,115 \%$ \\
\hline$(2020)$ & & UE27 7 & {$[0,03 \%-0,157 \%]^{7}$} & & & & {$[1,607 \%-4,115 \%]^{7}$} \\
\hline \multicolumn{8}{|c|}{${ }^{1}$ En miles de millones de euros de 2011. No se indica cuál es la medida de bienestar escogida. } \\
\hline \multicolumn{8}{|c|}{2 Separa calificados y no calificados, aunque el cambio es numéricamente igual en ambos, a un decimal. } \\
\hline \multicolumn{8}{|c|}{$\begin{array}{l}{ }^{3} \text { En miles de millones de USD de 2011. El trabajo proporciona la cifra en USD y se ha empleado un tipo de cambio de 0,719 euros/USD. Son variacio- } \\
\text { nes equivalentes. }\end{array}$} \\
\hline \multicolumn{8}{|c|}{${ }^{4}$ UE27 incluye el Reino Unido, pero no Países Bajos. } \\
\hline \multicolumn{8}{|c|}{${ }^{5}$ Variables en términos reales sectoriales. Flujos comerciales entre UE27 y Mercosur. } \\
\hline \multicolumn{8}{|c|}{$\begin{array}{l}6 \text { UE16 incluye Austria, Bélgica, Chequia, Alemania, Dinamarca, Francia, Grecia, Hungría, Irlanda, Italia, Luxemburgo, Países Bajos, Polonia, Portugal, } \\
\text { Eslovaquia y Suecia. }\end{array}$} \\
\hline \multicolumn{8}{|c|}{${ }^{7}$ UE27 incluye el Reino Unido pero no Austria. } \\
\hline \multicolumn{8}{|l|}{ Fuente: elaboración propia. } \\
\hline
\end{tabular}


simulación consiste exclusivamente en reducir los aranceles a todos los sectores. Eso incluye los sectores considerados tanto por la UE como por Mercosur como sensibles. En realidad, el comercio de los productos de estos sectores no se va a liberalizar como el del resto de bienes no sensibles, lo cual impide que se vayan a obtener las ganancias derivadas de la reducción de barreras comerciales sobre estos bienes sensibles. Por tanto, la comparación que se puede hacer en términos de PIB es que con reglas específicas para productos sensibles las ganancias son muy bajas (inferiores al $1 \%$ del PIB) en relación con un acuerdo que integrara una eliminación de barreras a estos productos sensibles, cuyo resultado sería más próximo al que obtienen Sanguinet y Alvim (2020).

Los efectos sobre el salario real también muestran unos efectos relativamente pequeños. Cuando el trabajo se asume móvil entre sectores, siempre se observa un aumento del salario real, pero también en niveles relativamente bajos, inferiores al 0,4\%, siendo del $0,1 \%$ en el único trabajo que estima esta variable para el caso español. Cuando el estudio asume especificidad del factor trabajo por sectores, los efectos son cuantitativamente mayores, como es previsible, y estos oscilan entre descensos de hasta el $0,7 \%$ y aumentos del $1,5 \%$.

En cuanto a los flujos comerciales, estas variables son lógicamente las que muestran un mayor cambio cuantitativo. Hay que resaltar que las estimaciones de Timini y Viani (2020) y de Sinabell, Grübler y Reiter (2020) permiten comparar a España con otros países de la UE. $Y$ en ambos trabajos se obtiene que España sería el país que, para los países incluidos, experimenta mayor repercusión en sus flujos comerciales. En el caso del estudio de Timini y Viani (2020), el aumento estimado de importaciones para España es del 1,29\%, mientras que el de exportaciones es del 1,44\%. $Y$ del estudio de Sinabell, Grübler y Reiter (2020), cuyos resultados son mayores para todos los países en relación con los del anterior trabajo, el nivel de exportaciones en España subiría un $4,115 \%$.

Por último, se puede indicar que, aunque sean metodologías diferentes y su comparación haya que interpretarla con cautela, el trabajo de equilibrio general de London School of Economics Consulting (2020) proporciona unos efectos cuantitativos para el conjunto de la UE inferiores a las estimaciones con ecuación de gravedad de Timini y Viani (2020) y de Sinabell, Grübler y Reiter (2020).

\section{Conclusiones}

El largo proceso de negociación entre la Unión Europea y los países de Mercosur ha ido generando un volumen amplio de estudios que han abordado los potenciales efectos económicos de un acuerdo de libre comercio. La complejidad de las políticas simuladas ha ido creciendo, ya que el acuerdo final no se limita a una eliminación o reducción de aranceles, sino que muchos aspectos vinculados a barreras no arancelarias están presentes en el acuerdo y en muchos de los estudios aquí recogidos. El proceso de negociación culminó en 2019.

En estos momentos se dispone ya de un cierto número de estudios que permite concluir que los efectos del último acuerdo, tanto para la Unión Europea como para España, serían cuantitativamente pequeños. La existencia de un número amplio de productos considerados sensibles por las dos partes negociadoras ha hecho que el proceso de apertura comercial no sea grande con ellos y que las ganancias $D$ 
que se podrían derivar de un acuerdo más ambicioso no puedan ser alcanzadas. Así, el acuerdo explotaría, solo ligeramente, el potencial de las ventajas comparativas de la UE y España en manufacturas y servicios, y el de Mercosur en productos agroalimentarios, aunque a pesar de ello se prevén ganancias para todos.

\section{Bibliografía}

Aguiar, A., Narayanan, B., y McDougall, R. (2016). An overview of the GTAP 9 data base. Journal of Global Economic Analysis, 1(1), 181-208.

Balassa, B. (1965). Trade liberalisation and "revealed" comparative advantage. The Manchester School, 33(2), 99-123.

Baltensperger, M., y Dadush, U. (2019). The European Union-Mercosur Free Trade Agreement: prospects and risks. Bruegel Policy Contribution, (11).

Bebek, U. G. (2011). Consistency of the proposed additive measures of revealed comparative advantage. Economics Bulletin, 31(3), 2491-2499.

Boulanger, P., Dudu, H., Ferrari, E., Himics, M., and M'barek, R. (2016). Cumulative economic impact of future trade agreements on EU agriculture.EUR28206EN.https://doi.org/10.2788/194880

Boyer, I., y Schuschny, A.R. (2010). Quantitative assessment of a free trade agreement between MERCOSUR and the European Union. Serie Estudios estadísticos y prospectivos CEPAL (69). https://repositorio.cepal.org/handle/11362/4774

Burrel, A., Ferrari, E., González Mellado, A., Himics, M., Michalek, J., Shrestha, S., y Van Doorslaer, B. (2011). Potential EU-MERCOSUR Free Trade Agreement: Impact Assessment. Joint Research Centre-EU, Luxembourg Publications Office of the European Union. https://ec.europa.eu/jrc/ en/publication/reference-reports/potential-eu- mercosur-free-trade-agreement-impact-assessment

Carrico, C., van Berkum, S., Tabeau, A., Jager, J., y Plaisier, N. (2020). Impacts of the EU-Mercosur trade agreement on the Dutch economy. Wageningen Economic Research Report, (2020065). https://doi.org/10.18174/539424

Copenhagen Economics (2011). Assessment of barriers to trade and investment between the EU and Mercosur. Economic Impact Assessment by request of European Commission. Comisión Europea DG Trade. https://trade.ec.europa.eu/doclib/docs/2011/november/tradoc_148370.pdf

Diao, X., Díaz-Bonilla, E., y Robinson, S. (2003). Scenarios for trade integration in the Americas. Économie International, (94-95), 33-52.

Estrades, C. (2012). Is MERCOSUR's External Agenda Pro-Poor? An Assessment of the European Union-MERCOSUR Free-Trade Agreement on Poverty in Uruguay Applying MIRAGE. (IFPRI Discussion paper 01219). International Food Policy Research Institute (IFPRI). http:// ebrary.ifpri.org/cdm/ref/collection/p15738coll2/ $\mathrm{id} / 127216$

Flôres, R.G., y Watanuki, M. (2008). Integration options for Mercosur: A quantitative analysis by the AMIDA model. (Working Paper 36). Institute for the Integration of Latin America and the Caribbean (INTAL), Inter-American Development Bank.

Gómez Gómez-Plana, A. (2005). Simulación de políticas económicas: los modelos de equilibrio general aplicado. Cuadernos Económicos de ICE, (69), 197-218.

Grieger, G. (2019). The trade pillar of the EU-Mercosur Association Agreement. European Parliamentary Research Service. https://www.europarl.europa.eu/RegData/etudes/BRIE/2019/ 640138/EPRS_BRI(2019)640138_EN.pdf

Harrison, G.W., Rutherford, T.F., Tarr, D.G., y Gurgel, A. (2003). Regional, Multilateral and Unilateral Trade Policies of MERCOSUR for Growth $\triangleright$ 
and Poverty Reduction in Brazil. (Policy Research Working Paper 3051). World Bank, Development Research Group.

Kirkpatrick, C., Franz, J., George, C., Hennelin, B., Hinojosa, L., Kutas, G., Maff, A., Nelson, P., Olsen, J., y Ryder, M. (2006). Trade Sustainability Impact Assessment (SIA) of the association agreement under negotiation between the European Community and Mercosur. Inception Report (September 2006) carried out for the European Commission. https://trade.ec.europa.eu/ doclib/docs/2006/september/tradoc_129267.pdf

Kirkpatrick, C., George, C., Doctor, M., Hinojosa, L., Grainger, A., Iwanow, T., Murinde, V., y Regina, P. (2009). Trade Sustainability Impact Assessment (SIA) of the association agreement under negotiation between the European Community and Mercosur. Final Report (March 2009), carried out for the European Commission. https://trade. ec.europa.eu/doclib/docs/2009/april/tradoc_142921.pdf

London School of Economics Consulting (2018). Sustainability Impact Assessment in support of association agreement negotiations between the European Union and Mercosur. Inception Report. https://trade.ec.europa.eu/doclib/docs/ 2018/march/tradoc_156631.pdf

London School of Economics Consulting (2020). Sustainability Impact Assessment in Support of the Association Agreement Negotiations between the European Union and Mercosur. Draft Final Report. https://trade.ec.europa.eu/doclib/ docs/2020/july/tradoc_158892.pdf

Martínez Serrano, J. A. (2020). La crisis del multilateralismo. Información Comercial Española ICE. Revista de Economía, (913), 17-32.

Philippidis, G., Resano, H., y Sanjuán, A.I. (2014). Shifting Armington trade preferences: A re-examination of the Mercosur-EU negotiations. Economic Modelling, 40, 21-32. https://doi.or$\mathrm{g} / 10.1016 / \mathrm{j}$. econmod.2014.03.016
Piketty, M.G., Weissleder, L., Meirelles de Souza FiIho, H., Batalha, M., Adenäuer, M., y Becker, A. (2009). Assessing agricultural trade policies options with the European Union: a Brazilian perspective. Agricultural Economics, 40(4), 447-457.

Sanahuja, J.A., y Rodríguez, J.D. (2019). Veinte años de negociaciones Unión Europea-Mercosur: Del interregionalismo a la crisis de la globalización. (Documentos de trabajo 13-2019). Fundación Carolina. https://www.fundacioncarolina.es/wp-content/uploads/2019/09/DT_FC_13.pdf

Sanguinet, E., y Alvim, A. (2020). Effects of EU-Mercosur trade agreement on bilateral trade: the role of Brexit. (MPRA Paper 103010). https:// mpra.ub.uni-muenchen.de/103010/1/MPRA_paper_102995.pdf

Sinabell, F., Grübler J., y Reiter, O. (2020). Implication of the EU-Mercosur Association Agreement for Austria. A Preliminary Assessment. FIW research report.

Timini, J., y Viani, F. (2020). A highway across the Atlantic? Trade and welfare effects of the EU-Mercosur Agreement. (Documento de Trabajo 2023). Banco de España. https://www.bde. es/f/webbde/SES/Secciones/Publicaciones/PublicacionesSeriadas/DocumentosTrabajo/20/Files/dt2023e.pdf

Weissleder, L., Adenäuer, M., y Heckelei, T. (2008). Impact assessment of trade liberalisation between EU and Mercosur Countries. [Paper prepared for presentation at the 107th EAAE Seminar «Modeling of Agricultural and Rural Development Policies»]. Sevilla, España; 29 de enero-1 de febrero de 2008. https://www.researchgate.net/publication/23509026_Impact_ Assessment_of_Trade_Liberalisation_Between_ EU_and_Mercosur_Countries

Yu, R., Cai, J., y Leung, P. (2009). The normalized revealed comparative advantage index. The $A n$ nals of Regional Science, 43(1), 267-282. https:// doi.org/10.1007/s00168-008-0213-3 
